\title{
THE KERNEL OF AN IRREDUCIBLE MAP
}

\author{
HENNING KRAUSE
}

(Communicated by Maurice Auslander)

\begin{abstract}
Let $0 \rightarrow A \rightarrow B \stackrel{g}{\rightarrow} C \rightarrow 0$ be a short exact sequence in the category of finitely generated modules over an artin algebra. Suppose also that the map $g$ is irreducible. Following a conjecture of Brenner, we discuss the property of the indecomposable module $A$ to be the starting term of an almost split sequence with indecomposable middle term.
\end{abstract}

Let $\Lambda$ be an artin algebra over a commutative artin ring $R$ and denote by $\bmod \Lambda$ the category of finitely generated $\Lambda$-modules. In [AR2] Auslander and Reiten introduced the notion of an irreducible morphism. They define a morphism $g: B \rightarrow C$ in $\bmod \Lambda$ to be irreducible if $g$ is neither a split mono nor a split epi and if, for any factorization $g=g_{2} g_{1}$, either $g_{1}$ is a split mono or $g_{2}$ is a split epi. The following numerical invariant is defined for an indecomposable noninjective $\Lambda$-module $A$. Let $0 \rightarrow A \rightarrow E \rightarrow \operatorname{Tr} D A \rightarrow 0$ be an almost split sequence. Then $\alpha(\operatorname{Tr} D A)$ denotes the number $n$ of summands in a decomposition of $E=\bigsqcup_{i=1}^{n} E_{i}$ into indecomposable modules. It is obvious from the definition that an irreducible map is either a mono or an epi. Moreover the kernel $A$ of an irreducible epi is indecomposable [AR2]. Now Brenner conjectured some years ago that such a kernel satisfies $\alpha(\operatorname{Tr} D A)=1$. The main purpose of this note is to establish the following result, which has been obtained independently also by Brenner in [B].

Theorem. Let $0 \rightarrow A \rightarrow B \stackrel{g}{\rightarrow} C \rightarrow 0$ be an exact sequence in $\bmod \Lambda$ which is not almost split. Suppose that one of the modules $B$ and $C$ is indecomposable and that $g$ is irreducible. Then $\alpha(\operatorname{Tr} D A)=1$ if $A$ is not simple.

Let $n>1$ be a natural number. The following example shows that $\alpha(\operatorname{Tr} D A)$ $=n$ can occur for simple $A$. Let $k$ be a field and consider the algebra $\Lambda_{n}=$ $k\left[x_{1}, \ldots, x_{n}\right] /\left(x_{1}, \ldots, x_{n}\right)^{2}$. Denote by $S$ the unique simple $\Lambda_{n}$-module and let $I$ be its injective envelope. Then the exact sequence $0 \rightarrow S \rightarrow I \rightarrow I / S \rightarrow 0$ satisfies the assumptions of the Theorem and $\alpha(\operatorname{Tr} D S)=n$.

It is possible to describe in some detail the irreducible map $g$ if $\alpha(\operatorname{Tr} D A)>$ 1. These results are collected in Corollary 3.4 and for self-injective algebras in Corollary 3.5. The obvious question-which indecomposable noninjective modules $A$ with $\alpha(\operatorname{Tr} D A)=1$ actually occur as kernel of a nontrivial irreducible map-is answered in Proposition 3.7.

Received by the editors January 22, 1992 and, in revised form, August 26, 1992.

1991 Mathematics Subject Classification. Primary 16G70. 
As a consequence of the Theorem one obtains the existence of almost split sequences with indecomposable middle term for any nonsemisimple artin algebra. Different proofs have been published by Auslander and Reiten [AR2] (for algebras of finite representation type), Martínez-Villa [M], and Butler and Ringel [BR]. We point out that in fact $\bmod \Lambda$ has as many nonisomorphic almost split sequences with indecomposable middle term as there are nonisomorphic indecomposables if $\Lambda$ is of strongly unbounded representation type (i.e., for some $n \in \mathbf{N}$ there are infinitely many nonisomorphic objects in $\bmod \Lambda$ of length $n$ ) [K].

\section{IRREDUCIBLE MAPS AND THEIR CONTEXT}

We start with some notation and recall definitions. For $\Lambda$-modules $X$ and $Y$ we often write $(X, Y)$ instead of $\operatorname{Hom}_{\Lambda}(X, Y)$. Denote by $P(X, Y) \subseteq$ $\operatorname{Hom}_{\Lambda}(X, Y)$ the maps which factor through a projective module. The elements of $\underline{\operatorname{Hom}}_{\Lambda}(X, Y)=\operatorname{Hom}_{\Lambda}(X, Y) / P(X, Y)$ form together with the induced composition the morphisms of the category $\underline{\bmod } \Lambda$, which has the same objects as $\bmod \Lambda$. Dually $\overline{\bmod } \Lambda$ is defined modulo the injective modules.

The usual duality of $\bmod R$ is given by $D=\operatorname{Hom}_{R}(, I)$, where $I$ is an injective envelope of $R / \operatorname{rad} R$. The functor $D$ induces a duality between $\bmod \Lambda$ and $\bmod \Lambda^{\mathrm{op}}$. The transpose is denoted by $\operatorname{Tr}$ and defines a duality between $\underline{\bmod } \Lambda$ and $\underline{\bmod } \Lambda^{\text {op }}$.

We use the classical notation and compose maps from right to left. In that way the group $\operatorname{Hom}_{\Lambda}(X, Y)$ becomes an $\operatorname{End}_{\Lambda}(Y)$ - $\operatorname{End}_{\Lambda}(X)$-bimodule.

Let $C$ be a $\Lambda$-module. A map $g: B \rightarrow C$ is defined to be right almost split if it is not a split epi and if every map $X \rightarrow C$ which is not a split epi, factors through $g$. Note that $C$ is indecomposable if there exists a right almost split map $B \rightarrow C$. If in addition $g b=g$ for an endomorphism $b \in$ $\operatorname{End}_{\Lambda}(B)$ implies that $b$ is an isomorphism, then the map $g$ is refered to be minimal right almost split. It is convenient to call a nonzero map $g: B \rightarrow C$ partial right almost split if there exists a nonzero map $g^{\prime}: B^{\prime} \rightarrow C$ such that $\left[g g^{\prime}\right]: B \amalg B^{\prime} \rightarrow C$ is minimal right almost split. Let $0 \rightarrow A \rightarrow B \stackrel{g}{\rightarrow} C \rightarrow 0$ be an exact sequence. The sequence is by definition almost split if $g$ is minimal right almost split. We call such a sequence partial right almost split if $g$ is partial right almost split. Of course there is also the dual notion of left almost split maps, including their variations. Almost split maps and sequences were introduced by Auslander and Reiten. We refer to their papers for existence proofs and properties.

The following well-known characterization of an irreducible map as well as its dual pendant will be used throughout this paper without any further reference. The proof is straightforward (modulo the existence of almost split sequences) and may be found in [AR2].

Proposition 1.1. For a nonsplit exact sequence $0 \rightarrow A \stackrel{f}{\rightarrow} B \stackrel{g}{\rightarrow} C \rightarrow 0$ the following are equivalent:

(i) The map $g$ is irreducible.

(ii) Given a map $a: A \rightarrow X$ there exists either a map $s: X \rightarrow B$ such that $f=s a$ or a map $t: B \rightarrow X$ such that $a=t f$.

If in addition $C$ is indecomposable, then the above is equivalent to: 
(iii) There exists a map $g^{\prime}: B^{\prime} \rightarrow C$ such that $\left[g g^{\prime}\right]: B \amalg B^{\prime} \rightarrow C$ is minimal right almost split.

For convenience we restate the definition of a partial right almost split sequence using Proposition 1.1.

Lemma 1.2. For an exact sequence $0 \rightarrow A \rightarrow B \stackrel{g}{\rightarrow} C \rightarrow 0$ the following are equivalent:

(i) The sequence is partial right almost split.

(ii) The sequence is not almost split, $C$ is indecomposable, and $g$ is irreducible

It is interesting to note that a partial right almost split sequence is not uniquely (up to isomorphism) determined by its end terms. Consider, for example, the algebra $\Lambda=k\langle x, y\rangle /\left(x^{2}, y^{2}, x y\right)$ over a field $k$. Denote by $S$ the unique simple $\Lambda$-module and let $I$ be its injective envelope. The module $I / S$ decomposes, say $I / S \cong S \amalg A$, and $\operatorname{Ext}_{\Lambda}^{1}(I, A)$ contains two nonisomorphic partial right almost split sequences. However, a partial right almost split sequence $x \in \operatorname{Ext}_{\Lambda}^{1}(C, A)$ is unique up to isomorphism, if $\operatorname{End}_{\Lambda}(C)$ is a division ring.

Let $M$ be a module over an arbitrary ring. We call a submodule $W \subseteq M$ a waist of $M$ if $U \subseteq W$ or $W \subseteq U$ for every submodule $U \subseteq M$. Note that, in contrast to the usual definition, the trivial submodules are also waist.

The following proposition which is also due to Auslander and Reiten is an immediate consequence of characterization (ii) in Proposition 1.1.

Proposition 1.3. Let $0 \rightarrow A \stackrel{f}{\rightarrow} B \stackrel{g}{\rightarrow} C \rightarrow 0$ be exact and suppose that $g$ is irreducible.

(a) The module $A$ is indecomposable.

(b) The $\operatorname{End}_{\Lambda}(X)$-module $\operatorname{Im}(f, X) \subseteq \operatorname{Hom}_{\Lambda}(A, X)$ is a waist for every $\Lambda$-module $X$.

\section{THE BIMODULE ASSOCIATED WITH AN IRREDUCIBLE MAP}

Given an irreducible epi $B \rightarrow C$ with kernel $A$, an analysis of the $\operatorname{End}_{\Lambda}(C)$ $\operatorname{End}_{\Lambda}(\operatorname{Tr} D A)$-bimodule $\operatorname{Hom}_{\Lambda}(\operatorname{Tr} D A, C)$ will be of importance for the proof of the Theorem. The corresponding result is Proposition 2.6, but we need some preparations. Let us first recall a very useful result of Auslander [A, III, Theorem 4.1], which lies at the heart of the theory.

Proposition 2.1. Let $x: 0 \rightarrow A \stackrel{f}{\rightarrow} B \stackrel{g}{\rightarrow} C \rightarrow 0$ be exact. For every $\Lambda$-module $X$ there is an isomorphism $\operatorname{Coker}(f, D \operatorname{Tr} X) \cong D \operatorname{Coker}(X, g)$ which is functorial in $X$ and $x$.

For a proof we have to refer to $[A]$. The following two consequences are essentially contained in [AR1] and [A], respectively.

Proposition 2.2. Let $X$ and $C$ be $\Lambda$-modules. There are isomorphisms

$$
\alpha: \operatorname{Ext}_{\Lambda}^{1}(C, D \operatorname{Tr} X) \stackrel{\sim}{\rightarrow} D \underline{\operatorname{Hom}}_{\Lambda}(X, C)
$$


and

$$
\beta: \operatorname{Ext}_{\Lambda}^{1}(C, D \operatorname{Tr} X) \stackrel{\sim}{\rightarrow} D \overline{\operatorname{Hom}}_{\Lambda}(D \operatorname{Tr} X, D \operatorname{Tr} C)
$$

which are functorial in $X$ and $C$ such that for $x \in \operatorname{Ext}_{\Lambda}^{1}(C, D \operatorname{Tr} X)$ the following diagram commutes (where I denotes the injective envelope of $R / \operatorname{rad} R$ ):

$$
\begin{array}{cr}
\underline{\operatorname{Hom}}_{\Lambda}(X, C) & \stackrel{\alpha(x)}{\longrightarrow} I \\
l \downarrow D \operatorname{Tr} & \| \\
\overline{\operatorname{Hom}}_{\Lambda}(D \operatorname{Tr} X, D \operatorname{Tr} C) \stackrel{\beta(x)}{\longrightarrow} I
\end{array}
$$

Proof. Choose an exact sequence $0 \rightarrow K \stackrel{f}{\rightarrow} P \stackrel{g}{\rightarrow} C \rightarrow 0$ with projective $P$. Then $\operatorname{Coker}(f)=,\operatorname{Ext}_{\Lambda}^{1}(C$,$) and \operatorname{Coker}(, g)=\underline{\operatorname{Hom}}_{\Lambda}(, C)$. From Proposition 2.1 we obtain an isomorphism $\operatorname{Ext}_{\Lambda}^{1}(C, D \operatorname{Tr} X) \stackrel{\sim}{\rightarrow} D \underline{\operatorname{Hom}}_{\Lambda}(X, C)$, which is functorial in $X$ and $C$. This proves the existence of $\alpha$. The isomorphism $\beta$ is obtained by composing $\alpha$ with the inverse of the functorial isomorphism $D(D \operatorname{Tr}): D \overline{\operatorname{Hom}}_{\Lambda}(D \operatorname{Tr} X, D \operatorname{Tr} C) \stackrel{\sim}{\rightarrow} D \underline{\operatorname{Hom}}_{\Lambda}(X, C)$. The commutativity follows immediately from the construction of $\beta$.

Lemma 2.3. Let $x: 0 \rightarrow D \operatorname{Tr} X \stackrel{f}{\rightarrow} B \stackrel{g}{\rightarrow} C \rightarrow 0$ be exact and denote by $\alpha_{x}=$ $\alpha(x)$ and $\beta_{x}=\beta(x)$ the maps corresponding to $x$ under the isomorphisms of Proposition 2.2.

(a) Let $U \subseteq \underline{\operatorname{Hom}}_{\Lambda}(X, C)$ be an $\overline{\operatorname{End}}_{\Lambda}(X)$-submodule. Then $U \subseteq \operatorname{Ker} \alpha_{x}$ iff $U \subseteq \operatorname{Im}(X, \underline{g})$.

(b) Let $U \subseteq \overline{\operatorname{Hom}}_{\Lambda}(D \operatorname{Tr} X, D \operatorname{Tr} C)$ be an $\overline{\operatorname{End}}_{\Lambda}(D \operatorname{Tr} C)$-submodule. Then $U \subseteq \operatorname{Ker} \beta_{x}$ iff $U \subseteq \operatorname{Im}(\bar{f}, D \operatorname{Tr} C)$.

Proof. (a) Since the isomorphism $\alpha$ of Proposition 2.2 is functorial in $C$, we have for $u \in \underline{\operatorname{Hom}}_{\Lambda}(X, C)$ that $\alpha_{x} \operatorname{Hom}_{\Lambda}(X, u)=\alpha\left(\operatorname{Ext}_{\Lambda}^{1}(u, D \operatorname{Tr} X)(x)\right)=0$ iff $u \in \operatorname{Im}(X, \underline{g})$. Therefore $\alpha_{x}(u)=\left(\alpha_{x} \underline{\operatorname{Hom}}_{\Lambda}(X, u)\right)\left(\operatorname{id}_{X}\right)=0$ for $u \in$ $\operatorname{Im}(X, \underline{g})$ and hence $\operatorname{Im}(X, \underline{g}) \subseteq \operatorname{Ker} \alpha_{x}$. Now suppose $U \nsubseteq \operatorname{Im}(X, \underline{g})$, say $u \in U \backslash \operatorname{Im}(X, g)$. Then $\alpha_{x}(u t)=\left(\alpha_{x} \operatorname{Hom}_{\Lambda}(X, u)\right)(t) \neq 0$ for some $t \in$ End $_{\Lambda}(X)$ and therefore $U \nsubseteq \operatorname{Ker} \alpha_{x}$, since $U$ is a submodule. This finishes the proof.

(b) Dual to (a).

Lemma 2.4. Let $0 \rightarrow D \operatorname{Tr} X \stackrel{f}{\rightarrow} B \stackrel{g}{\rightarrow} C \rightarrow 0$ be partial right almost split. There is an $\operatorname{End}_{\Lambda}(D \operatorname{Tr} C)$-submodule $U \subseteq \operatorname{Hom}_{\Lambda}(D \operatorname{Tr} X, D \operatorname{Tr} C)$ such that $U$ is a waist and $\operatorname{rad} U=\operatorname{Im}(f, D \operatorname{Tr} C) \subseteq U$ is a maximal submodule.

Proof. We infer from the isomorphism $(D \operatorname{Tr} X, D \operatorname{Tr} C) / \operatorname{Im}(f, D \operatorname{Tr} C) \cong$ $D((C, C) / \operatorname{Im}(C, g))$ of Proposition 2.1 that the socle of $(D \operatorname{Tr} X, D \operatorname{Tr} C) /$ $\operatorname{Im}(f, D \operatorname{Tr} C)$ over $\operatorname{End}_{\Lambda}(D \operatorname{Tr} C)$ is simple, since $C$ is indecomposable. Now define $U$ by

$$
U / \operatorname{Im}(f, D \operatorname{Tr} C)=\operatorname{soc}((D \operatorname{Tr} X, D \operatorname{Tr} C) / \operatorname{Im}(f, D \operatorname{Tr} C)) .
$$

The properties of $U$ stated above follow from the fact that $\operatorname{Im}(f, D \operatorname{Tr} C)$ is a waist by Proposition 1.3. 
Lemma 2.5. Let $x: 0 \rightarrow D \operatorname{Tr} X \stackrel{f}{\rightarrow} B \stackrel{g}{\rightarrow} C \rightarrow 0$ be partial right almost split. There is an $\underline{\text { End }}_{\Lambda}(C)$-submodule $V \subseteq \underline{\operatorname{Hom}}_{\Lambda}(X, C)$ with the following properties:

(i) $V$ and $\operatorname{rad} V$ are waists, and $\operatorname{rad} V \subseteq V$ is a maximal submodule.

(ii) $\operatorname{rad} V \subseteq \operatorname{Im}(X, \underline{g})$ and $V \nsubseteq \operatorname{Im}(X, \underline{g})$.

Proof. We fix the isomorphism

$$
\tau=D \operatorname{Tr}: \underline{\operatorname{Hom}}_{\Lambda}(X, C) \stackrel{\sim}{\rightarrow} \overline{\operatorname{Hom}}_{\Lambda}(D \operatorname{Tr} X, D \operatorname{Tr} C)
$$

which is $\underline{\text { End }}_{\Lambda}(C)$-linear, if we consider the ring isomorphism $D \operatorname{Tr}$ : $\underline{\text { End }}_{\Lambda}(C)$ $\stackrel{\sim}{\rightarrow} \overline{\operatorname{End}}_{\Lambda}(\overline{D T r} C)$ as identification. Now define $V=\tau^{-1}(\bar{U})$, where $\bar{U}$ denotes the image of the $\operatorname{End}_{\Lambda}(D \operatorname{Tr} C)$-module $U$ of the previous lemma under the canonical map $\operatorname{Hom}_{\Lambda}(D \operatorname{Tr} X, D \operatorname{Tr} C) \rightarrow \overline{\operatorname{Hom}}_{\Lambda}(D \operatorname{Tr} X, D \operatorname{Tr} C)$. This is an End $_{\Lambda}(C)$-submodule of $\underline{\operatorname{Hom}}_{\Lambda}(X, C)$. Let us verify each of the properties stated above.

(i) Follows immediately from Lemma 2.4 since $\tau$ is an isomorphism.

(ii) Denote by $\alpha_{x}$ and $\beta_{x}$ the elements of $D \underline{\operatorname{Hom}}_{\Lambda}(X, C)$ and $D \overline{\operatorname{Hom}}_{\Lambda}(D \operatorname{Tr} X, D \operatorname{Tr} C)$, respectively, which correspond to $x$ under the isomorphisms of Proposition 2.2. Recall that $\alpha_{x}=\beta_{x} \tau$. First we show $\operatorname{rad} V \subseteq$ $\operatorname{Im}(X, \underline{g})$. By Lemma $2.3 \operatorname{rad} \bar{U}=\operatorname{Im}(\bar{f}, D \operatorname{Tr} C) \subseteq \operatorname{Ker} \beta_{x}$. Therefore $\operatorname{rad} V=\tau^{-1}(\operatorname{rad} \bar{U}) \subseteq \operatorname{Ker} \alpha_{x}$. Now $\operatorname{rad} V$ is also an $\underline{\operatorname{End}}_{\Lambda}(X)$-submodule of $\underline{\operatorname{Hom}}_{\Lambda}(X, C)$ since it is a waist over $\underline{\operatorname{End}}_{\Lambda}(C)$ by part (i). Therefore $\operatorname{rad} V \subseteq \operatorname{Im}(X, \underline{g})$ by Lemma 2.3. Following the same line of arguments, one obtains $V \nsubseteq \operatorname{Im}(X, \underline{g})$ from the fact that $\bar{U} \nsubseteq \operatorname{Im}(\bar{f}, D \operatorname{Tr} X)$.

Proposition 2.6. Let $0 \rightarrow D \operatorname{Tr} X \rightarrow B \stackrel{g}{\rightarrow} C \rightarrow 0$ be partial right almost split. There exists an element $v \in \underline{\operatorname{Hom}}_{\Lambda}(X, C) \backslash \operatorname{Im}(X, \underline{g})$ such that for every $u \in$ $\underline{\operatorname{Hom}}_{\Lambda}(X, C) \backslash \operatorname{Im}(X, \underline{g})$ there is some $c \in \underline{\operatorname{End}}_{\Lambda}(C)$ with $v=c u$.

Proof. We apply Lemma 2.5. Choose any $v \in V \backslash \operatorname{Im}(X, \underline{g})$. Then

$$
u \notin \operatorname{Im}(X, \underline{g}) \text { implies } V \subseteq \underline{\operatorname{End}}_{\Lambda}(C) u
$$

and therefore $v=c u$ for some $c \in \underline{\operatorname{End}}_{\Lambda}(C)$.

\section{Proof of the Theorem}

We proceed in four steps. Proposition 3.1 may be regarded as a first approximation of the final Theorem. I learned it from S. Brenner. It will be combined with our previous results from the second section to obtain Proposition 3.2. The next step is a direct application of that proposition, which provides a proof of the Theorem for most situations. The last step is the proof of Proposition 3.6, which covers the remaining case. The latter proof gives a rather different approach and is independent from the second section, whereas Proposition 3.1 is also used.

Proposition 3.1. Let $x: 0 \rightarrow A \stackrel{f}{\rightarrow} B \stackrel{g}{\rightarrow} C \rightarrow 0$ be an exact sequence which is not almost split. Suppose that $B$ or $C$ is indecomposable and that $g$ is irreducible. Suppose also that

$$
0 \rightarrow A \stackrel{\left[\begin{array}{c}
a \\
a^{\prime}
\end{array}\right]}{\rightarrow} E \coprod E^{\prime} \stackrel{\left[b b^{\prime}\right]}{\rightarrow} \operatorname{Tr} D A \rightarrow 0
$$


is an almost split sequence with $E \neq 0$.

(a) There exists a map $s: E \rightarrow B$ such that $f=s a$.

(b) There exists a map $u: \operatorname{Tr} D A \rightarrow C$ such that $u b^{\prime}=0$ and $u \notin$ $\operatorname{Im}(\operatorname{Tr} D A, g)$.

Proof. (a) Assume there is no $s$ with $f=s a$. We seek a contradiction. Since $g$ is irreducible there exists $t: B \rightarrow E$ such that $a=t f$ and $t$ is a split epi because $a$ is irreducible. We consider two cases:

If $B$ is indecomposable, then the map $t$ is an iso. Therefore we obtain $f=t^{-1} a$, which contradicts our initial assumption.

Now suppose that $C$ is indecomposable. Let $\left.0 \rightarrow D \operatorname{Tr} C \rightarrow B \amalg B^{\prime[g} g^{\prime}\right]$ $C \rightarrow 0$ be almost split. Note that $g^{\prime} \neq 0$ since $x$ is not almost split. Then there is the following commutative diagram:

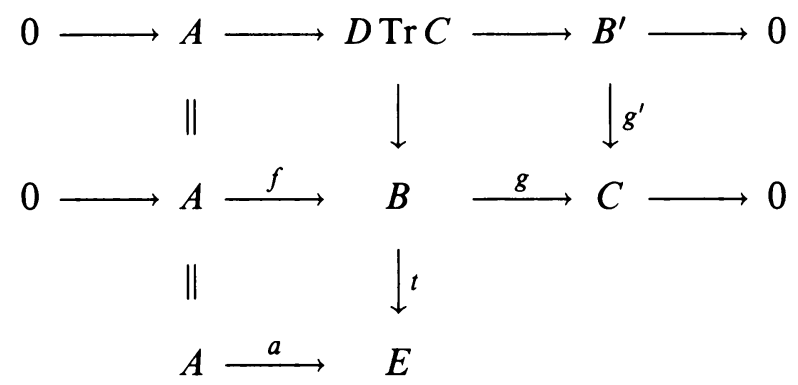

Neither $A \rightarrow D \operatorname{Tr} C$ nor the composition $D \operatorname{Tr} C \rightarrow B \rightarrow E$ splits. This contradicts the fact that $a$ is irreducible, and therefore the proof of (a) is complete.

(b) This an immediate consequence of part (a) since we have a commutative diagram of the following form:

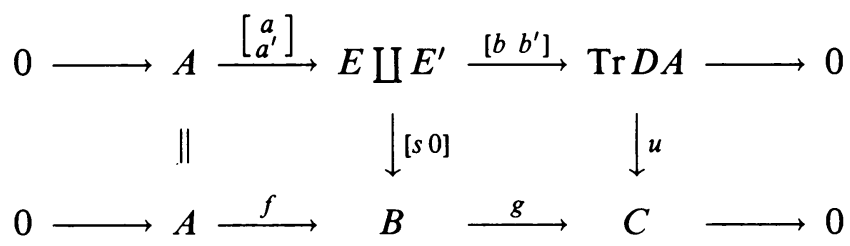

Proposition 3.2. Let $0 \rightarrow A \stackrel{f}{\rightarrow} B \stackrel{g}{\rightarrow} C \rightarrow 0$ be partial right almost split, and suppose that $\alpha(\operatorname{Tr} D A)>1$.

(a) There is a factorization $f=p a$ such that $a$ is irreducible and $p$ factors through a projective module.

(b) The module $A$ is simple.

Proof. (a) Let $0 \rightarrow A \rightarrow E \amalg E^{\prime} \stackrel{\left[b b^{\prime}\right]}{\rightarrow} \operatorname{Tr} D A \rightarrow 0$ be almost split with nonzero $E$ and $E^{\prime}$. Applying Proposition 3.1, we obtain maps $u, u^{\prime} \in$ $\mathrm{Hom}_{\Lambda}(\operatorname{Tr} D A, C) \backslash \operatorname{Im}(\operatorname{Tr} D A, g)$ such that $u b^{\prime}=0=u^{\prime} b$. By Proposition 2.6 there exist $c, c^{\prime} \in \operatorname{End}_{\Lambda}(C)$ such that $v=c u \notin \operatorname{Im}(\operatorname{Tr} D A, g)$ and $c u-c^{\prime} u^{\prime} \in P(\operatorname{Tr} D A, C)$. We have $v b^{\prime}=0$ and $v b=\left(c u-c^{\prime} u^{\prime}\right) b \in P(E, C)$. Therefore there exists $p \in P(E, B)$ such that $v b=g p$. Now consider the 
following pullback diagram:

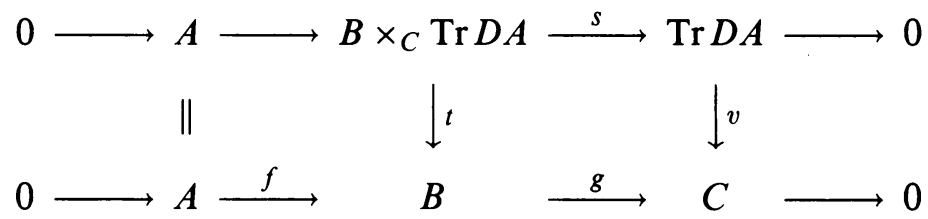

Since $v\left[b b^{\prime}\right]=g[p 0]$, there exists $i: E \amalg E^{\prime} \rightarrow B \times{ }_{C} \operatorname{Tr} D A$ such that $\left[b b^{\prime}\right]=$ $s i$ and $[q 0]=t i$. The map $i$ is a split mono because $\left[\begin{array}{ll}b & b^{\prime}\end{array}\right]$ is irreducible and $s$ does not split. Moreover $i$ is an iso since $E \amalg E^{\prime}$ and $B \times{ }_{C} \operatorname{Tr} D A$ have same length. Therefore we obtain the following diagram, which finishes the proof of part $(\mathrm{a})$ :

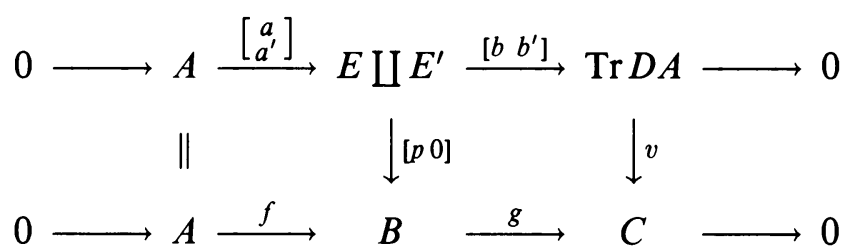

(b) Let $S$ be a simple submodule of $A$ and let $s: A \rightarrow A / S$ be the canonical map. Since $s$ is not a mono we obtain from the characterization (ii) in Proposition 1.1 a map $r$ which makes the following diagram commute:

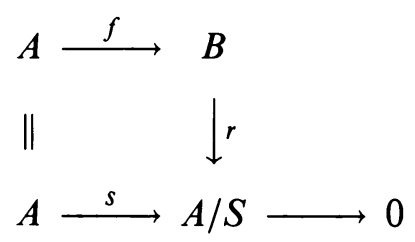

We use the factorization $f=p a$ of part (a). The composition $r p$ factors through a projective and this gives a map $t$ such that $r p=s t$. Hence $s=$ $r p a=s t a$, which implies $s=0$ since $t a$ is nilpotent. We conclude that $A=S$ is simple.

Lemma 3.3. Let $0 \rightarrow A \rightarrow B \stackrel{g}{\rightarrow} C \rightarrow 0$ be exact. Suppose that $B$ is indecomposable noninjective and that $g$ is irreducible. Then there exists a partial right almost split sequence $0 \rightarrow A \rightarrow B^{\prime} \stackrel{g^{\prime}}{\rightarrow} C^{\prime} \rightarrow 0$.

Proof. There is an almost split sequence $0 \rightarrow B \stackrel{\left[\begin{array}{c}a \\ a^{\prime}\end{array}\right]}{\rightarrow} E \amalg E^{\prime} \stackrel{\left[b b^{\prime}\right.}{\rightarrow} \operatorname{Tr} D B \rightarrow$ 0 such that $a=g$. The kernel of the partial right almost split map $b^{\prime}$ is isomorphic to $A$. Therefore we may choose $g^{\prime}=b^{\prime}$.

Proof of the Theorem. Let $0 \rightarrow A \rightarrow B \stackrel{g}{\rightarrow} C \rightarrow 0$ be as in the statement of the Theorem. We want to show that $\alpha(\operatorname{Tr} D A)=1$ if $A$ is not simple. This follows from Proposition 3.2 combined with Lemma 3.3 for indecomposable $C$ and for indecomposable noninjective $B$. The remaining case of an indecomposable injective $B$ is covered by Proposition 3.6.

Corollary 3.4. Let $0 \rightarrow A \stackrel{f}{\rightarrow} B \stackrel{g}{\rightarrow} C \rightarrow 0$ be an exact sequence in $\bmod \Lambda$ which is not almost split. Suppose that one of the modules $B$ and $C$ is indecomposable 
and that $g$ is irreducible. If $\alpha(\operatorname{Tr} D A)>1$, then the following three cases are possible:

(i) $A$ is torsionless (submodule of a projective) and f factors through a projective module.

(ii) $A$ is torsionless, $B$ is isomorphic to an indecomposable summand of $\operatorname{rad} P$ for some indecomposable projective $P$, and $C$ decomposes.

(iii) $A$ is not torsionless, $B$ is indecomposable injective, and $C$ decomposes.

Proof. We use part (a) of Proposition 3.2. If $C$ is indecomposable, then we obtain case (i). Now suppose that $C$ decomposes and $B$ is indecomposable. If $B$ is injective we obtain case (i) or (iii). Otherwise there is an almost split sequence

$$
0 \rightarrow B \stackrel{\left[\begin{array}{l}
g \\
g^{\prime}
\end{array}\right]}{\rightarrow} C \coprod C^{\prime} \rightarrow \operatorname{Tr} D B \rightarrow 0
$$

which gives the following diagram:

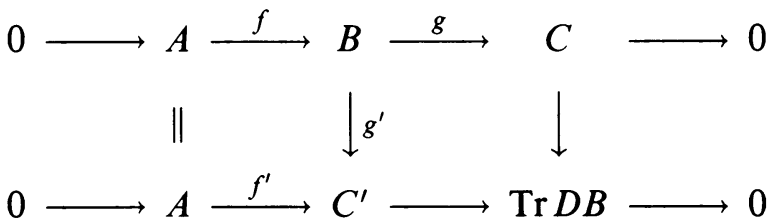

By Proposition $3.2 f^{\prime}=q p$ for two maps $p: A \rightarrow P$ and $q: P \rightarrow C^{\prime}$ where $P$ is projective. In particular $A$ is torsionless. We claim that $C^{\prime}$ is projective, if the map $f$ allows no factorization through $P$. This would lead to case (ii), since $g^{\prime}$ is irreducible and therefore a mono onto a summand of $\operatorname{rad} C^{\prime}$ (see [AR2]). To show that $C^{\prime}$ is projective we consider a map $p^{\prime}: B \rightarrow P$ such that $p=p^{\prime} f$, which exists by our assumption that $f$ does not factor through $p$. Therefore $f^{\prime}=q p^{\prime} f$. It is not hard to see that $q p^{\prime}$ needs to be irreducible. The map $p^{\prime}$ is not a spit mono, again since $f$ does not factor through $P$. Therefore $q$ is a split epi and $C^{\prime}$ is projective.

Corollary 3.5. Let $\Lambda$ be a self-injective artin algebra, and let $0 \rightarrow A \rightarrow B \stackrel{g}{\rightarrow}$ $C \rightarrow 0$ be an exact sequence in $\bmod \Lambda$, which is not almost split. Suppose that one of the modules $B$ and $C$ is indecomposable and that $g$ is irreducible. Then the following conditions are equivalent for a natural number $n>1$ :

(i) $\alpha(\operatorname{Tr} D A)=n$.

(ii) $A$ is simple and $\operatorname{rad} P / \operatorname{soc} P$ decomposes into $n$ summands, where $P$ denotes the projective cover of $A$.

(iii) $A$ is simple and $\operatorname{rad} I / \operatorname{soc} I$ decomposes into $n$ summands, where I denotes the injective envelope of $A$.

Moreover, if $A$ satisfies these conditions, then $B$ is either indecomposable projective or isomorphic to the radical of some indecomposable projective module.

Proof. Recall that for self-injective $\Lambda$ the syzygy functor $\Omega: \underline{\bmod \Lambda} \rightarrow \underline{\bmod \Lambda}$ is an equivalence. Therefore the conditions (i), (ii), and (iii) are equivalent for simple $A$, since we have $\operatorname{rad} P / \operatorname{soc} P \cong \Omega(E)$ and $\Omega(\operatorname{rad} I / \operatorname{soc} I) \cong D \operatorname{Tr} E$, where $E$ denotes the middle term of an almost split sequence $0 \rightarrow A \rightarrow E \rightarrow$ $\operatorname{Tr} D A \rightarrow 0$.

Now assume that $\alpha(\operatorname{Tr} D A)=n>1$. The proof is complete if we show that $A$ is simple and that $B \cong P$ or $B \cong \operatorname{rad} P$ for some indecomposable projective 
$P$. First consider the case that $C$ is indecomposable. Therefore Proposition 3.2 applies. Thus $A$ is simple and we obtain an indecomposable projective module $P$ such that $p a \neq 0$ for two maps $a: A \rightarrow P$ and $p: P \rightarrow B$. The map $a$ is an injective envelope, which implies the existence of $b: B \rightarrow P$ with $a=b p a$. We conclude that $b p$ is an isomorphism and therefore $B$ has an injective summand isomorphic to $P$. Consequently $C \cong P / \operatorname{soc} P$, since up to isomorphism $P \rightarrow$ $P / \operatorname{soc} P$ is the unique irreducible map starting in $P$ (see [AR2]). A length argument shows that actually $B \cong P$. If $C$ is not indecomposable, then $B$ is indecomposable and noninjective by our assumptions. The construction of Lemma 3.3 combined with our previous argument shows that $B \cong \operatorname{rad} P$ for some indecompsable projective module $P$.

To finish the proof of the Theorem we reproduce some arguments of MartinezVilla (see [M]).

Proposition 3.6. Let $x: 0 \rightarrow A \rightarrow B \stackrel{g}{\rightarrow} C \rightarrow 0$ be an exact sequence. Suppose that $A$ is nonsimple, $B$ is injective, and $g$ is irreducible.

(a) If $0 \rightarrow A \rightarrow E \amalg E^{\prime} \stackrel{\left[b b^{\prime}\right]}{\rightarrow} \operatorname{Tr} D A \rightarrow 0$ is an almost split sequence, then $b$ or $b^{\prime}$ is an epi.

(b) If $x$ is not almost split and $B$ or $C$ is indecomposable, then $\alpha(\operatorname{Tr} D A)$ $=1$.

Proof. (a) Let $S$ be a simple submodule of $A$ and choose any nonzero $\underline{s} \in$ $\underline{\operatorname{Hom}}_{\Lambda}(\operatorname{Tr} D S, \operatorname{Tr} D A) \cong \overline{\operatorname{Hom}}_{\Lambda}(S, A) \neq 0$. We claim that $s$ is an epi. This immediately implies that $b$ or $b^{\prime}$ is an epi, since there exists a map $\left[\begin{array}{c}r \\ r^{\prime}\end{array}\right]: \operatorname{Tr} D S \rightarrow$ $E \amalg E^{\prime}$ such that $s=b r+b^{\prime} r^{\prime}$ and $\underline{b r} \neq 0$ or $\underline{b^{\prime} r^{\prime}} \neq 0$.

Now let $X$ be the cokernel of $s$ and fix an exact sequence $\operatorname{Tr} D S \stackrel{s}{\rightarrow} \operatorname{Tr} D A \stackrel{t}{\rightarrow}$ $X \rightarrow 0$. We apply $D \operatorname{Tr}$ and obtain $\bar{u}=D \operatorname{Tr} \underline{s}, \bar{v}=D \operatorname{Tr} \underline{t}$ with $\overline{v u}=0$. Therefore $v u=p i$ for some map $p: I \rightarrow D \operatorname{Tr} X$ if $i: S \rightarrow I$ denotes an injective envelope of $S$. Now assume $v u \neq 0$. Then $v u$ is a mono and we obtain a map $q: D \operatorname{Tr} X \rightarrow I$ such that $i=q v u$. Therefore $I$ is a summand of $D \operatorname{Tr} X$, which is impossible. We conclude that $v u=0$. This implies $\operatorname{Ker} v \neq 0$ since $u \neq 0$. Therefore $v$ factors through $B$ since $g$ is irreducible. Hence $\bar{v}=0$ and $\underline{t}=0$. Finally this implies $t=0$ since $\operatorname{Tr} D A$ has no projective summand. Thus we have shown that $X=\operatorname{Coker} s=0$.

(b) Combine part (a) with Proposition 3.1.

Let us now answer the question of when a module $A$ satisfying $\alpha(\operatorname{Tr} D A)=1$ is the kernel of a nontrivial irreducible map.

Proposition 3.7. Let $0 \rightarrow A \rightarrow E \rightarrow \operatorname{Tr} D A \rightarrow 0$ be an almost split sequence with indecomposable $E$. Then the following are equivalent:

(i) The module $E$ is not injective.

(ii) There exists a partial right almost split sequence $0 \rightarrow A \rightarrow B \rightarrow C \rightarrow 0$.

(iii) There exists an exact sequence $0 \rightarrow A \rightarrow B \stackrel{g}{\rightarrow} C \rightarrow 0$ which is not almost split such that one of $B$ and $C$ is indecomposable and $g$ is irreducible.

Proof. That (i) implies (ii) is a special case of Lemma 3.3, and (ii) trivially implies (iii). It remains to show that (i) is a consequence of (iii). Therefore 
let $0 \rightarrow A \stackrel{f}{\rightarrow} B \stackrel{g}{\rightarrow} C \rightarrow 0$ be an exact sequence as in (iii). We assume $E$ to be injective and seek a contradiction. The map $f$ factors through the left almost split map $A \rightarrow E$, which we denote by $a$. On the other hand, $a$ factors through $f$ since $E$ is injective. We obtain the following commutative diagram with exact rows:

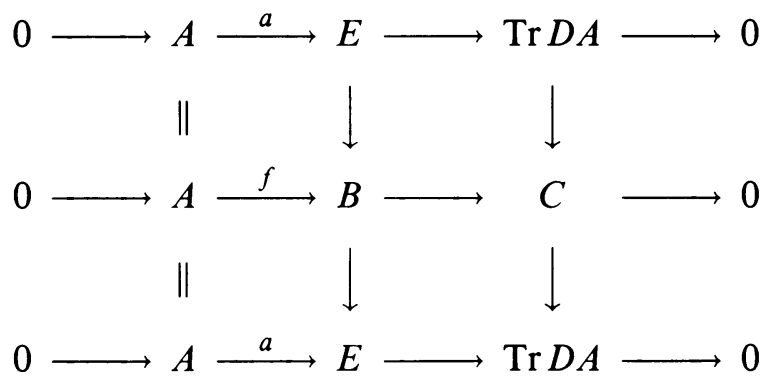

The vertical compositions are isomorphisms since $a$ is an injective envelope of $A$. In fact each of the vertical maps is an isomorphism since $B$ or $C$ is indecompsable. But this contradicts the fact that the sequence $0 \rightarrow A \stackrel{f}{\rightarrow} B \stackrel{g}{\rightarrow}$ $C \rightarrow 0$ is not almost split.

Remark. If an almost split sequence $0 \rightarrow A \rightarrow E \rightarrow \operatorname{Tr} D A \rightarrow 0$ has an injective middle term, then $\operatorname{Tr} D A$ is simple (see [AR1]).

\section{ACKNOWLEDGMENT}

This paper originated from discussions with Sheila Brenner. It was completed after I got introduced to functors determined by objects by Maurice Auslander and his collaborators. I would like to thank all of them. The author is supported by a DFG postdoctoral grant.

\section{REFERENCES}

[A] M. Auslander, Functors and morphisms determined by objects, Representation Theory of Algebras (R. Gordon, ed.) (Proc. Conf., Philadelphia, 1976), Dekker, New York, 1978, pp. 1-244.

[AR1] M. Auslander and I. Reiten, Representation theory of artin algebras. III, Comm. Algebra 3 (1975), 239-294.

[AR2] _ Representation theory of artin algebras. IV, Comm. Algebra 5 (1977), 443-518.

[B] S. Brenner, On kernels of irreducible maps, preprint, 1991.

[BR] M. C. R. Butler and C. M. Ringel, Auslander-Reiten sequences with few middle terms and applications to string algebras, Comm. Algebra 15 (1987), 145-179.

[K] $\mathrm{H}$. Krause, On the number of almost split sequences with indecomposable middle term, Bull. London Math. Soc. (to appear).

[M] R. Martínez-Villa, Almost projective modules and almost split sequences with indecomposable middle term, Comm. Algebra 8 (1980), 1123-1150.

Department of Mathematics, Brandeis University, Waltham, Massachusetts 02254 Current address: Fakultät für Mathematik, Universität Bielefeld, 33501 Bielefeld, Germany E-mail address: henning@mathematik. uni-bielefeld.de 